\title{
Consumption and drinking frequency of alcoholic beverage among women in Ghana: a cross-sectional study
}

\author{
Anthony Mwinilanaa Tampah-Naah ${ }^{*}$ and Samuel Twumasi Amoah
}

\begin{abstract}
Background: The consumption and drinking frequency of alcoholic beverage are identified with various individual factors. The aim of this study was to identify background characteristics of women associated with the consumption and drinking frequency of alcoholic beverage.

Methods: Data was extracted from the 2008 Ghana Demographic and Health Survey. The data consisted of women's (aged 15-49 years) background characteristics and their reported history of consumption and drinking frequency of alcoholic beverage. A weighted sample size of 4916 women was used in the present study. Binary logistic regression and multinomial logistic regression were applied to examine the independent association between the covariates and the consumption and drinking frequency of alcoholic beverage respectively.
\end{abstract}

Results: Out of the 4916 women that were included in the study, 17.5\% consumed alcoholic beverage in the past week. Factors that were found to be associated with women who consumed alcoholic beverage in a binary logistic regression model were age (15-19 years up to 45-49 years), region (Central, Greater Accra, Volta, Ashanti, Northern, Upper East, and Upper West), ethnicity (Ga or Dangme, Mole-Dagbani, Grussi, Gruma or Mande), wealth quintile (middle), and employment status [past 12 months] (those employed). In the multinomial logistic regression model, drinking frequency of alcoholic beverage was associated with women in the Central (none), Greater Accra Region (none and 4 or more times), Eastern (none and 2-3 times), Brong Ahafo (none), Upper East (none), those who attained primary education (4 or more times), Ga/Dangme ethnic group (none), those of middle wealth quintile (none), and those employed (4 or more times).

Conclusions: In the country, about 2 in 10 women consume alcoholic beverage and the drinking frequency of alcoholic beverage varied among women in the country. Hence, the maintenance of moderate alcoholic beverage consumption among women, where applicable, should be encouraged.

Keywords: Consumption, Drinking frequency, Alcoholic beverage, Women, Ghana

\section{Background}

Alcoholic beverage consumption is a global phenomenon which is considered as a public health priority worldwide [1,2]. According to Lim et al. [3], alcohol consumption moved from being the eighth highest ranked risk factor in 1990 to the fifth highest ranked risk factor in 2010. The mere consumption of alcoholic beverage by any person may not predispose them to health or social consequences. It is rather the quantity of alcoholic beverage

\footnotetext{
* Correspondence: tonytampah@yahoo.com

Department of Environment and Resource Studies, University for Development Studies, Wa Campus, Wa, Ghana
}

consumed and the drinking frequency that, importantly, can predict the likely health effects or other socially related consequences they encounter [4-6].

One segment of the alcohol consumption debate that has attracted enormous attention in most literature has been the recommended daily alcohol consumption $[7,8]$. In the alcohol consumption discourse, guidelines are unclear as to the preferred quantity to consume. In one epidemiological research, heavy or at risk drinking for women is defined as more than three drinks a day and for men as more than four drinks in a day $[9,10]$. Also, the Dietary Guidelines for Americans describe moderate 
alcohol consumption as drinking up to one drink per day for women and up to two drinks for men [11]. In South Africa, women are advised not to drink more than two 'standard drinks' a day and men are not to drink more than three 'standard drinks' a day. According to this guideline, 'standard drink' means 1 can of beer $(340 \mathrm{ml}), 1$ tot of spirits, 1 glass of white or dry red wine $(125 \mathrm{ml})$ or 1 small glass of sherry $(60 \mathrm{ml})$ [12]. Although a defined level of alcohol consumption is unclear in the literature, alcohol consumption may be influenced by a person's age, gender, body weight, height, economic status and health status.

The consumption of alcoholic beverage is associated with certain health benefits and risks. Numerous studies have revealed that consuming alcoholic beverage moderately have some health benefits to the consumer; such as sharp reduction in heart disease risk [9], low mortality [13,14] and reduced risk of stroke by about half [15]. The benefits of moderate alcoholic beverage consumption to the heart and others have, therefore, been accepted by many researchers $[16,17]$. Consuming alcoholic beverage moderately is, hence, linked with better health status and longevity than either abstaining or abusing it [18]. However, the consumption of alcohol moderately, per its general definition, may not be beneficial to everyone; it is advisable to personally and medically examine oneself to determine how moderately to consume alcoholic beverage. On the other hand, heavy consumption of alcoholic beverage can be a prelude to diseases such as cardiovascular diseases [19], indigestion and gastrointestinal ulcers [20], obesity, metabolic syndrome and glucose intolerance [21-23]; reproductive health problems [24] and chronic kidney disease, induced hypertension and liver diseases [25-28].

Globally, it is estimated that $55 \%$ of women have never consumed alcoholic beverage [29]. Despite the high abstinence rates of alcoholic beverage consumption, there is a growing concern that there are high risk patterns of drinking among women who have consumed alcoholic beverage in the past year in many low-and middle-income countries [30]. In Ghana, among current drinkers, about 4\% of women were heavy alcoholic beverage consumers [31].

Unfortunately, there exist only a draft national alcohol policy intended to be passed by government to guide the consumption, importation, manufacturing, and marketing of alcoholic beverages [32]. This has culminated in paucity and inconsistent information on the consumption of alcoholic beverage in the past decades [33]. Consequently, the control of alcoholic beverage sales and advertisements in the media remains a major challenge in the country [34]. With the absence of a national alcohol policy, the country has continuously witnessed the proliferation of alcoholic beverages with regular advertisements on the media especially televisions, radio stations and on wide bill boards [35], with women playing major roles in most of these alcoholic beverage advertisements [36,37].
The consumption of either foreign alcoholic beverages (such as wines and whiskeys) or locally produced alcoholic beverages such as pito, liqueurs (Alomo Bitters, Kasapreko, Striker, Pusher or Ginseng) and gin (Akpeteshie) among women can lead to undesirable outcomes such as unplanned pregnancy [38,39]. Agoabasa [40] noted that women resort to the consumption of alcoholic beverage as a way to cope with stress and anxiety in society. Despite these persistent claims, to the best of our knowledge, there exist a dearth of research in Ghana which presents empirical analysis of the characteristics that bears out the alcoholic beverage consumption and drinking frequency of women. Based on this literature gab, the present study aimed to analyze the characteristics that underpin alcoholic beverage consumption and drinking frequency among women in Ghana; using data from the 2008 Ghana Demographic and Health Survey (GDHS) [33].

\section{Methods}

\section{Study area}

Ghana is located in West Africa and has a total land area of 238, 537 square kilometers [33]. The country's population is about $24,658,823$ people. This consists of 12 , 633, 978 (51.2\%) females and 12, 024, 845 males (48.8\%); translating to a sex ratio of 95 males to 100 females [41].

\section{Study design and sample}

The study employed a cross-sectional approach using data set from the 2008 Ghana Demographic and Health Survey (GDHS). The individual (women) data set was specifically used for the data analysis. The GDHSs are national-level population and health surveys conducted in Ghana as part of the global Demographic and Health Survey programme. These surveys are designed to provide information to monitor the population and health situation in Ghana [33].

The 2008 GDHS used a two-stage sample based on the 2000 Population and Housing Census to produce separate estimates for key indicators for each of the ten regions in Ghana. Each household selected for the 2008 GDHS was eligible for interview with a household questionnaire. On the part of women, the survey involved a total of 4,916 women aged 15-49 years and the response rate to the survey questionnaire yielded a rate of 97 percent.

All women $(4,916)$ who took part in the survey were considered for analysis in the present study. Sample size was weighted using the relevant weight $(\mathrm{w}=\mathrm{v005})$ as recommended by MeasureDHS. To validate the data, frequencies and percentages of the various characteristics of women were recalculated. And the results were compared to the figures in the 2008 GDHS report for consistency. The usage of this data was approved by MeasureDHS (Demographic and Health Survey) on June 29, 2011through their website http://www.measuredhs.com. 


\section{Dependent variables}

The present study considered two dependent variables: Alcoholic beverage consumption and drinking frequency of alcoholic beverage. The first dependent variable, alcoholic beverage consumption, was operationalized as whether a woman (aged 15-49 years old) has consumed, at least one drink of any type of alcoholic beverage in the past week preceding the survey. The term "drink" in the definition can be a bottle of beer/stout, a glass of wine, a shot of liquor/a mixed drink with liquor, or a calabash of pito/palm wine. This was guided by the question (as captured in the 2008 GDHS questionnaire), "Do you consume alcoholic beverage?" The expected response to this question was either "Yes" or "No"; these were adopted by the present study.

The second dependent variable, drinking frequency of alcoholic beverage, was defined by the present study as "the frequency (once, 2-3 times, 4 times or more, or not at all) of consuming alcoholic beverage within the past week preceding the survey by a woman of the age cohort 15-49 years old". The words "drinking" and "consuming" have been used interchangeably in the present study. This definition was informed by a follow up question to the alcoholic beverage consumption; thus the first dependent variable. Those women who responded in affirmative to the alcohol consumption question were further asked "How frequently they had consumed alcohol during the past week: ..." (GSS 2009, p.60).

\section{Independent variables}

The independent variables (background characteristics of women) that were used include: age, marital status, residence, region, education, ethnicity, wealth, and employment. Some of the variables were recoded while others were adopted as reported in the 2008 GDHS:

- Age of women (15-49 years old) were coded as: 15-19, 20-24, 25-29, 30-34, 35-39, 40-44, and 45-49.

- Marital status of women was recoded into three groups: Never married, married/cohabiting and divorced/separated/widowed.

- Residence was categorized as urban and rural as captured in the 2008 GDHS.

- Region, all the ten regions in the country were considered. These are Western, Central, Greater Accra, Volta, Eastern, Ashanti, Brong Ahafo, Northern, Upper East and Upper West.

- Education was reported in terms of a woman's educational level. This variable was recoded as no education, primary and secondary/higher.

- Ethnicity, categories were recoded into five groups: Akan, Ga/Dangme, Ewe/Guan, Mole-Dagbani and Grussi/Gruma/Mande/others.
- Wealth quintile coding was adopted as captured in the 2008 GDHS report: Poor, middle and rich.

- Employment (past 12 months) was captured as not employed and employed.

Independent variables' categories that had smaller number of frequencies were collapsed or regrouped with similar categories to form larger categories. For instance, marital status, education, ethnicity and wealth quintile categories were regrouped.

\section{Data analysis}

Descriptive statistics were used to present the background characteristics of the women. Also, chi-square test of independence was performed to test for individual independent association $(p<0.05)$ between the independent and dependent variables, and proportions were presented in terms of the women who consumed alcohol. With the drinking frequency of alcohol, summaries were presented in frequencies and proportions.

Further analyses were applied in this study: Binary logistic regression and multinomial logistic regression. All background variables were considered for the binary logistic regression since all were statistically significant $(\mathrm{p}<0.05)$. Only the variable age was not considered for the multinomial logistic regression.

The binary logistic regression was applied to generate a model for women who consumed alcoholic beverage in the past week. This form of analysis was applied since the dependent variable had dichotomous responses; with responses coded as "Yes" = 1 (consume alcoholic beverage) and "No" = 0 (do not consume alcoholic beverage). Initially, unadjusted binary logistic regression model was generated and later adjusted model was built by controlling for all statistical significant $(\mathrm{p}<0.05)$ characteristics.

Similarly, all statistical significant $(\mathrm{p}<0.05)$ characteristics with the drinking frequency of alcohol (none, once, 2-3 times, and 4 times or more) were primarily used to build unadjusted models. Subsequently, adjusted models were generated by controlling for all significant $(\mathrm{p}<0.05)$ independent variables. Women that consumed alcoholic beverage once in the past week before the survey were used as the reference group because they reported most of consuming alcoholic beverage.

Both results of the binary logistic regression and multinomial logistic models were presented as odds ratio with their corresponding 95\% confidence intervals (CI). Data processing and statistical analysis were done using STATA version 11.

\section{Results}

Table 1 shows distribution of background characteristics of women that were considered in the study. The study revealed that the pass week alcoholic beverage consumption 
Table 1 Background characteristics of women $(\mathrm{N}=4916)$

\begin{tabular}{lll}
\hline Characteristics & $\mathbf{n}$ & \% \\
\hline Age & & \\
$15-19$ & 1025 & 20.8 \\
$20-24$ & 878 & 17.9 \\
$25-29$ & 832 & 16.9 \\
$30-34$ & 644 & 13.1 \\
$35-39$ & 638 & 13.0 \\
$40-44$ & 470 & 9.6 \\
$45-49$ & 429 & 8.7
\end{tabular}

Marital status*

Never married

Married/Cohabiting

2876

Divorced/Separated/Widowed

446

Residence

Urban

Rural

2533

Region

Western

Central

Greater Accra

Volta

Eastern

Ashanti

Brong Ahafo

1011

Northern

Upper East

253

Upper West

Education*

No education

1042

Primary

988

Secondary/Higher

2692

Ethnicity*

Akan

2493

Ga/Dangme

343

Ewe/Guan

Mole-Dagbani

755

795

528

Grussi/Gruma/Mande

Wealth

Poor

Middle

1683

979

Rich

Employment (past 12 months)

\begin{tabular}{lll} 
Not employed & 1240 & 25.2 \\
Employed & 3676 & 74.8 \\
\hline
\end{tabular}

$\mathrm{n}$ : frequency of variable, \%: percentage of frequency, ${ }^{*} \mathrm{~N}<4916$ due to missing responses. in the country was $17.5 \%$. From the distribution, there were proportional variations in the consumption of alcoholic beverage among the independent variables (Table 2).

All the independent variables were considered for generating the binary logistic regression model since all exhibited statistical significant $(p<0.05)$ association with the first dependent variable (alcoholic beverage consumption) (Table 2). Controlling for all variables, categories that were found to be associated with alcoholic beverage consumption among women in the binary logistic regression model were 15-19 years to 45-49 years, Central, Greater Accra, Volta, Ashanti, Northern, Upper East, and Upper West, Ga/Dangme, Mole-Dagbani, Grussi,/ Gruma /Mande, middle wealth quintile, and employed women. Women in the 45-49 years category were 4 times more likely to consume alcoholic beverage compared to women in the $15-19$ years category $(\mathrm{AOR}=4.42$; $95 \% \mathrm{CI}$ : $2.91,6.70)$. Women in the Upper West Region were about 12 times more likely to consume alcoholic beverage compared to women in the Western Region $(\mathrm{AOR}=11.98$; 95\% CI: 7.60, 18.90). Women of the Ga or Dangme ethnic group were more likely to consume alcoholic beverage compared to women of the Akan ethnic group (AOR = 1.66; 95\% CI: 1.21, 2.28). Likewise, women of the middle wealth quintile were less likely to consume alcoholic beverage compared to those in the poor wealth quintile $(\mathrm{AOR}=0.64 ; 95 \% \mathrm{CI}: 0.50,0.81)$. In terms of employment (past 12 months), women who were employed were more likely to consume alcoholic beverage compared to those who were not employed (AOR $=1.46$; 95\% CI: 1.16, 1.83).

Table 3 provides bivariate associations of the drinking frequency of alcoholic beverage among women. Out of the eight independent variables examined in the bivariate association, only age was not statistical significant $(\mathrm{p}<0.05)$ with drinking frequency of alcoholic beverage.

The multinomial logistic regression models are displayed on Table 4. After controlling for all significant $(\mathrm{p}<0.05)$ independent variables: region, education, ethnicity, wealth quintile and employment exhibited association with drinking frequency of alcoholic beverage. Women in the Central Region were 5 times more likely not to consume alcoholic beverage rather than once compared to women in the Western Region (AOR = $5.34,95 \% \mathrm{CI}=7.94,14.70)$. Likewise, women in the Eastern Region were found to be 3 times more likely to consume alcoholic beverage 2-3 times rather than once compared to those in the Western Region ( $\mathrm{AOR}=3.09$, $95 \% \mathrm{CI}=1.03$, 9.27). Similarly, women in the Greater Accra Region were 6 times more likely to consume alcoholic beverage 4 or more times rather than once compared to those in Western Region (AOR $=6.19$, 95\% $\mathrm{CI}=1.03$, 37.19). On education, women who had primary education were 2 times more likely to consume alcoholic beverage 4 or more times rather than once 
Table 2 Proportions and predictors of alcoholic beverage consumption among women

\begin{tabular}{|c|c|c|c|c|c|c|c|}
\hline \multirow[t]{2}{*}{ Characteristics } & \multicolumn{2}{|c|}{ Alcohol consumption } & \multirow[t]{2}{*}{ p-value } & \multicolumn{2}{|c|}{ Age adjusted } & \multicolumn{2}{|c|}{ Multivariate } \\
\hline & $\mathrm{n}=861$ & $\%=17.5$ & & COR & $95 \% \mathrm{Cl}$ & AOR & $95 \% \mathrm{Cl}$ \\
\hline Age & & & 0.000 & & & & \\
\hline $15-19$ & 68 & 6.6 & & 1 & & 1 & \\
\hline $20-24$ & 146 & 16.7 & & $2.40^{* * *}$ & $1.81,3.17$ & $2.40^{* * *}$ & $1.73,3.32$ \\
\hline $25-29$ & 137 & 16.5 & & $2.63^{* * *}$ & $1.98,3.48$ & $2.57^{* * *}$ & $1.78,3.72$ \\
\hline $30-34$ & 133 & 20.7 & & $3.11^{* * *}$ & $2.33,4.16$ & $2.99^{* * *}$ & $2.02,4.42$ \\
\hline $35-39$ & 162 & 25.4 & & $4.10^{* * *}$ & $3.09,5.43$ & $3.82^{* * *}$ & $2.59,5.65$ \\
\hline $40-44$ & 104 & 22.2 & & $3.51^{* * *}$ & $2.59,4.75$ & $2.98^{* * *}$ & $1.96,4.52$ \\
\hline $45-49$ & 111 & 26.0 & & $4.76^{* * *}$ & $3.51,6.43$ & $4.42^{* * *}$ & $2.91,6.70$ \\
\hline Marital status & & & 0.000 & & & & \\
\hline Never married & 175 & 11.0 & & 1 & & 1 & \\
\hline Married/Cohabiting & 569 & 19.8 & & $2.04^{* * *}$ & $1.71,2.43$ & 0.87 & $0.66,1.13$ \\
\hline Divorced/Separated/Widowed & 117 & 26.3 & & $2.85^{* * *}$ & $2.19,3.70$ & 1.34 & $0.94,1.70$ \\
\hline Residence & & & 0.000 & & & & \\
\hline Urban & 404 & 17.0 & & 1 & & 1 & \\
\hline Rural & 457 & 18.1 & & $1.40^{* * *}$ & $1.21,1.62$ & 1.23 & $0.98,1.55$ \\
\hline \multicolumn{8}{|l|}{ Region } \\
\hline Western & 48 & 10.7 & & 1 & & 1 & \\
\hline Central & 70 & 16.5 & & $2.04^{* *}$ & $1.33,3.14$ & $2.16^{* *}$ & $1.39,3.37$ \\
\hline Greater Accra & 223 & 26.2 & & $3.30^{* * *}$ & $2.29,4.75$ & $3.77^{* * *}$ & $2.49,5.69$ \\
\hline Volta & 105 & 24.3 & & $3.17^{* * *}$ & $2.15,4.68$ & $2.91^{* * *}$ & $1.86,4.54$ \\
\hline Eastern & 69 & 14.2 & & $1.65^{*}$ & $1.11,2.49$ & 1.48 & $0.97,2.27$ \\
\hline Ashanti & 131 & 13.0 & & 1.43 & $0.98,2.11$ & $1.69^{*}$ & $1.13,2.52$ \\
\hline Brong Ahafo & 37 & 8.6 & & 0.95 & $0.59,1.52$ & 1.06 & $0.65,1.71$ \\
\hline Northern & 71 & 15.2 & & $2.25^{* * *}$ & $1.52,3.33$ & $3.45^{* * *}$ & $2.21,5.41$ \\
\hline Upper East & 63 & 25.0 & & $3.21^{* * *}$ & $2.15,4.78$ & $5.62^{* * *}$ & $3.47,9.12$ \\
\hline Upper West & 46 & 37.3 & & $6.28^{* * *}$ & $4.32,9.11$ & $11.98^{* * *}$ & $7.60,18.90$ \\
\hline Education & & & 0.000 & & & & \\
\hline No education & 222 & 21.3 & & 1 & & 1 & \\
\hline Primary & 178 & 18.0 & & $0.67^{* * *}$ & $0.55,0.82$ & 0.95 & $0.76,1.8$ \\
\hline Secondary/Higher & 461 & 16.0 & & $0.53^{* * *}$ & $0.45,0.62$ & 0.85 & $0.69,1.06$ \\
\hline Ethnicity & & & 0.000 & & & & \\
\hline Akan & 359 & 14.4 & & 1 & & 1 & \\
\hline Ga/Dangme & 110 & 32.0 & & $2.51^{* * *}$ & $1.91,3.29$ & $1.66^{* *}$ & $1.21,2.28$ \\
\hline Ewe/Guan & 182 & 24.1 & & $1.86^{* * *}$ & $1.51,2.28$ & 1.20 & $0.91,1.59$ \\
\hline Mole-Dagbani & 120 & 15.1 & & $1.73^{* * *}$ & $1.43,2.08$ & $0.47^{* * *}$ & $0.34,0.67$ \\
\hline Grussi/Gruma/Mande & 89 & 16.9 & & $1.75^{* * *}$ & $1.40,2.18$ & $0.65^{* *}$ & $0.48,0.87$ \\
\hline Wealth & & & 0.000 & & & & \\
\hline Poor & 323 & 19.2 & & 1 & & 1 & \\
\hline Middle & 133 & 13.6 & & $0.51^{* * *}$ & $0.41,0.64$ & $0.64^{* * *}$ & $0.50,0.81$ \\
\hline Rich & 405 & 18.0 & & $0.69^{* * *}$ & $0.59,0.80$ & 0.87 & $0.67,1.13$ \\
\hline Employment (past 12 months) & & & 0.000 & & & & \\
\hline Not employed & 133 & 10.7 & & 1 & & 1 & \\
\hline Employed & 728 & 19.8 & & $2.16^{* * *}$ & 1.78 & $1.46^{* *}$ & $1.16,1.83$ \\
\hline
\end{tabular}


Table 3 Alcoholic beverage drinking frequency and associated factors among women

\begin{tabular}{|c|c|c|c|c|c|c|c|c|c|}
\hline \multirow[t]{3}{*}{ Characteristic } & \multicolumn{9}{|c|}{$\begin{array}{l}\text { Drinking patterns of drinking alcoholic } \\
\text { beverages (past } 7 \text { days) }(\mathrm{N}=861)\end{array}$} \\
\hline & \multicolumn{2}{|c|}{ None } & \multicolumn{2}{|c|}{ Once } & \multicolumn{2}{|c|}{$\begin{array}{l}2-3 \\
\text { times }\end{array}$} & \multicolumn{2}{|c|}{$\begin{array}{l}4 \text { timesor } \\
\text { more }\end{array}$} & \multirow[t]{2}{*}{ p-value } \\
\hline & $\mathrm{n}(\%)$ & & $n$ & $\%$ & $n$ & $\%$ & $n$ & $\%$ & \\
\hline Age & & & & & & & & & 0.063 \\
\hline 15-19 & 32 & 47.6 & 26 & 38.4 & 9 & 12.9 & 1 & 1.1 & \\
\hline $20-24$ & 62 & 42.1 & 59 & 40.7 & 20 & 13.7 & 5 & 3.5 & \\
\hline $25-29$ & 40 & 28.8 & 54 & 39.6 & 34 & 24.9 & 9 & 6.8 & \\
\hline $30-34$ & 46 & 34.6 & 48 & 33.5 & 28 & 20.7 & 15 & 11.6 & \\
\hline $35-39$ & 46 & 28.9 & 47 & 29.4 & 48 & 30.2 & 19 & 11.6 & \\
\hline $40-44$ & 29 & 28.4 & 42 & 41.1 & 25 & 24.3 & 6 & 6.2 & \\
\hline $45-49$ & 27 & 24.2 & 44 & 39.2 & 32 & 28.4 & 9 & 8.2 & \\
\hline
\end{tabular}

Marital status

$\begin{array}{lllllllll}\text { Never married } & 82 & 46.9 & 67 & 38.2 & 20 & 11.3 & 6 & 3.6\end{array}$

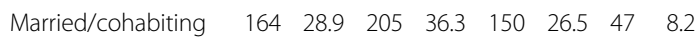

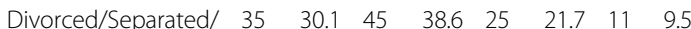
Widowed

Residence

Urban

Rural

Region

Western

Central

Greater Accra

Volta

Eastern

Ashanti

Brong Ahafo

Northern

Upper East

Upper West

Education

No education

Primary

Secondary/Higher

Ethnicity

Akan

Ga/Dangme

Ewe/Guan

Mole-Dagbani

Grussi/Gruma/

Mande

Wealth

Poor

Middle

Rich
$0.000^{*}$

$0.000^{*}$

$\begin{array}{llllllll}178 & 44.2 & 147 & 36.4 & 61 & 14.9 & 18 & 4.5\end{array}$

$\begin{array}{llllllll}103 & 22.7 & 170 & 37.5 & 135 & 29.7 & 46 & 10.1\end{array}$

$\begin{array}{llllllll}13 & 27.5 & 24 & 51.4 & 8 & 17.0 & 1 & 4.1\end{array}$

$\begin{array}{llllllll}38 & 55.3 & 15 & 21.9 & 9 & 13.5 & 6 & 9.3\end{array}$

$\begin{array}{llllllll}98 & 44.0 & 77 & 34.6 & 32 & 14.1 & 16 & 7.2\end{array}$

$\begin{array}{llllllll}12 & 11.3 & 57 & 54.8 & 29 & 27.8 & 6 & 6.1\end{array}$

$\begin{array}{llllllll}20 & 27.8 & 16 & 22.8 & 25 & 36.6 & 7 & 10.9\end{array}$

$\begin{array}{llllllll}55 & 42.9 & 43 & 33.3 & 26 & 20.5 & 4 & 3.2\end{array}$

$\begin{array}{llllllll}18 & 48.9 & 10 & 27.4 & 8 & 21.2 & 1 & 2.4\end{array}$

$\begin{array}{llllllll}18 & 25.2 & 25 & 35.1 & 20 & 28.3 & 8 & 11.4\end{array}$

$\begin{array}{llllllll}2 & 2.5 & 34 & 55.5 & 21 & 34.9 & 4 & 7.1\end{array}$

$\begin{array}{llllllll}6 & 13.5 & 15 & 32.7 & 16 & 35.9 & 8 & 17.9\end{array}$

$0.000^{*}$

$\begin{array}{llllllll}43 & 19.4 & 86 & 39.0 & 71 & 32.1 & 21 & 9.5\end{array}$

$\begin{array}{llllllll}44 & 24.9 & 62 & 35.6 & 45 & 25.4 & 25 & 14.2\end{array}$

$\begin{array}{llllllll}194 & 42.3 & 168 & 36.6 & 79 & 17.3 & 18 & 3.9\end{array}$

$0.000^{*}$

$\begin{array}{llllllll}166 & 46.1 & 110 & 30.7 & 63 & 17.6 & 20 & 5.6\end{array}$

$\begin{array}{llllllll}31 & 28.6 & 47 & 42.6 & 20 & 18.5 & 11 & 10.4\end{array}$

$\begin{array}{llllllll}49 & 26.9 & 75 & 41.2 & 46 & 25.5 & 12 & 6.4\end{array}$

$\begin{array}{llllllll}15 & 12.7 & 54 & 46.1 & 36 & 30.7 & 12 & 10.6\end{array}$

$\begin{array}{llllllll}20 & 23.0 & 30 & 34.3 & 29 & 33.1 & 8 & 9.7\end{array}$

$0.000^{*}$

$\begin{array}{llllllll}56 & 17.3 & 123 & 38.1 & 105 & 32.7 & 38 & 11.8\end{array}$

$\begin{array}{llllllll}56 & 42.2 & 40 & 30.3 & 25 & 18.8 & 12 & 8.7\end{array}$

$\begin{array}{llllllll}170 & 42.1 & 154 & 38.3 & 65 & 16.1 & 14 & 3.6\end{array}$
Table 3 Alcoholic beverage drinking frequency and associated factors among women (Continued)

\begin{tabular}{lllllllllll}
\hline $\begin{array}{l}\text { Employment } \\
\text { (past } 12 \text { months) }\end{array}$ & & & & & & & & & $0.037^{*}$ \\
Not employed & 53 & 40.7 & 51 & 38.6 & 23 & 17.4 & 4 & 3.2 & \\
Employed & 228 & 31.4 & 267 & 36.7 & 172 & 23.7 & 59 & 8.2 & \\
\hline
\end{tabular}

$\mathrm{n}$ : number of women, \%: percentage of women who consumed alcohol during the past week preceding the survey. ${ }^{*}$ Statistcally significant $(p<0.05)$. Note: Some frequencies do not correspond with frequencies in Table 2 due to missing responses.

compared to those with no education (AOR $=2.28,95 \%$ $\mathrm{CI}=1.17,4.42)$. In terms of ethnicity, women of the Ga or Dangme group were less likely not to consume alcoholic beverage compared to those of the Akan origin (AOR = $0.39,95 \% \mathrm{CI}=0.20,0.77$ ). With wealth quintile, women of middle wealth quintile were 2 times more likely not to consume alcoholic beverage rather than once compared to those in the poor wealth quintile ( $\mathrm{AOR}=2.26,95 \%$ $\mathrm{CI}=1.21,4.20)$. On employment, employed women were 3 times more likely to have consumed alcoholic beverage 4 or more times rather than once compared to those who were not employed $(\mathrm{AOR}=2.59,95 \% \mathrm{CI}=1.01,6.62)$.

\section{Discussion}

The present study used a nationwide dataset (2008 GDHS) to examine consumption and drinking frequency of alcoholic beverage in the country [33]. The results from the study evidenced that women of various categories in the country do consume alcoholic beverage. Although women in the country do consume alcoholic beverage, caution should be taken when examining the results since these may not indicate any form of risk drinking of alcoholic beverage. Also, it should be noted that the dataset used in the present study was based on a cross-sectional design and the survey's sample was drawn from women aged 15-49 years. Therefore, the purpose of this present study was to examine associated factors; first with women who consumed alcoholic beverage, and, second, with their drinking frequency of alcoholic beverage in the past week before the survey. It is worth noting that, there were proportional variations in the consumption and the drinking frequency of alcoholic beverage among women. The prevalence rate of alcoholic beverage consumption in the country was $17.5 \%$ [33]. For those women who consume alcoholic beverage once or several in a week are likely to have high volume of or low volume as compared to a woman who consumes one drink per week [42]. In a similar way, moderate consumers of alcoholic beverage could also be risk drinkers; considering how quickly they drink alcohol, their age or their health status. The discussion is focused on factors that were statistically significant after controlling for all independent variables both in the binary logistic regression and multinomial logistic regression models on alcohol consumption in the past week. 
Table 4 Association of alcohol drinking frequency with background characteristics of women $(\mathrm{N}=861)$

\begin{tabular}{|c|c|c|c|c|c|c|}
\hline \multirow[t]{2}{*}{ Characteristic } & \multicolumn{3}{|l|}{ Unadjusted } & \multicolumn{3}{|l|}{ Adjusted } \\
\hline & $\begin{array}{l}\text { None } \\
\text { (vs. Once) }\end{array}$ & $\begin{array}{l}2-3 \text { times } \\
\text { (vs. Once) }\end{array}$ & $\begin{array}{l}\geq 4 \text { times } \\
\text { (vs. Once) }\end{array}$ & $\begin{array}{l}\text { None } \\
\text { (vs. Once) }\end{array}$ & $\begin{array}{l}2-3 \text { times } \\
\text { (vs. Once) }\end{array}$ & $\begin{array}{l}\geq 4 \text { times } \\
\text { (vs. Once) }\end{array}$ \\
\hline & OR $(95 \% \mathrm{Cl})$ & OR $(95 \% \mathrm{Cl})$ & OR $(95 \% \mathrm{Cl})$ & AOR $(95 \% \mathrm{Cl})$ & AOR $(95 \% \mathrm{Cl})$ & AOR $(95 \% \mathrm{Cl})$ \\
\hline \multicolumn{7}{|l|}{ Marital status } \\
\hline Never married & 1 & 1 & 1 & 1 & 1 & 1 \\
\hline Married/cohabiting & $0.59(0.41,0.88)^{*}$ & $1.77(1.11,2.81)^{*}$ & $1.79(0.88,3.69)$ & $0.85(0.55,1.33)$ & $1.63(0.97,2.73)$ & $1.61(0.73,3.57)$ \\
\hline Divorced/Separated/Windowed & $0.64(0.37,1.12)$ & $1.35(0.72,2.55)$ & $1.80(0.72,4.51)$ & $0.61(0.33,1.12)$ & $1.30(0.67,2.54)$ & $1.76(0.65,4.73)$ \\
\hline \multicolumn{7}{|l|}{ Residence } \\
\hline Urban & 1 & 1 & 1 & 1 & 1 & 1 \\
\hline Rural & $0.42(0.30,0.58)^{*}$ & $2.05(1.41,2.96)^{*}$ & $2.48(1.38,4.45)^{*}$ & $0.86(0.51,1.45)$ & $1.53(0.88,2.66)$ & $1.5(0.62,4.02)$ \\
\hline \multicolumn{7}{|l|}{ Region } \\
\hline Western & 1 & 1 & 1 & 1 & 1 & 1 \\
\hline Central & $5.00(1.86,13.44)^{*}$ & $1.67(0.49,5.61)$ & $4.17(0.69,24.94)$ & $5.34(1.94,14.70)^{*}$ & $1.52(0.45,5.22)$ & $3.77(0.60,23.71)$ \\
\hline Greater Accra & $2.57(1.14,5.77)^{*}$ & $1.03(0.40,2.67)$ & $2.07(0.43,10.05)$ & $3.10(1.27,7.55)^{*}$ & $1.42(0.49,4.09)$ & $6.19(1.03,37.19)^{*}$ \\
\hline Volta & $0.49(0.19,1.26)$ & $1.45(0.57,3.68)$ & $0.91(0.16,5.07)$ & $0.55(0.18,1.66)$ & $0.92(0.29,2.91)$ & $0.92(0.12,6.92)$ \\
\hline Eastern & $2.22(0.85,5.83)$ & $3.19(1.15,8.91)^{*}$ & $3.89(0.71,21.19)$ & $3.36(1.20,9.36)^{*}$ & $3.09(1.03,9.27)^{*}$ & $3.89(0.64,23.59)$ \\
\hline Ashanti & $2.23(0.94,5.28)$ & $1.57(0.59,4.18)$ & $1.14(0.19,6.80)$ & $2.10(0.87,5.07)$ & $1.60(0.59,4.37)$ & $1.21(0.19,7.68)$ \\
\hline Brong Ahafo & $3.09(1.06,9.04)^{*}$ & $2.00(0.58,6.91)$ & $1.00(0.08,12.39)$ & $4.04(1.28,12.71)^{*}$ & $1.47(0.39,5.57)$ & $0.62(0.04,9.08)$ \\
\hline Northern & $1.21(0.48,3.07)$ & $2.50(0.95,6.55)$ & $4.67(0.96,22.79)$ & $1.96(0.56,6.92)$ & $0.98(0.27,3.59)$ & $3.21(0.39,26.59)$ \\
\hline Upper East & $0.10(0.03,0.39)^{*}$ & $1.29(0.51,3.31)$ & $0.93(0.17,5.16)$ & $0.18(0.36,0.94)^{*}$ & $0.84(0.23,3.11)$ & $0.53(0.06,5.11)$ \\
\hline Upper West & $0.69(0.28,1.65)$ & $2.66(1.09,6.49)^{*}$ & $4.75(1.04,21.72)^{*}$ & $1.31(0.38,4.48)$ & $1.73(0.49,6.08)$ & $2.45(0.31,19.61)$ \\
\hline \multicolumn{7}{|l|}{ Education } \\
\hline No education & 1 & 1 & 1 & 1 & 1 & 1 \\
\hline Primary & $1.83(1.11,3.03)^{*}$ & $0.92(0.59,1.42)$ & $1.63(0.93,2.87)$ & $1.17(0.65,2.11)$ & $1.09(0.66,1.81)$ & $2.28(1.17,4.42)^{*}$ \\
\hline Secondary/Higher & $3.20(2.16,4.76)^{*}$ & $0.63(0.44,0.92)^{*}$ & $0.44(0.24,0.81)^{*}$ & $1.39(0.79,2.46)$ & $1.01(0.60,1.69)$ & $0.82(0.36,1.86)$ \\
\hline \multicolumn{7}{|l|}{ Ethnicity } \\
\hline Akan & 1 & 1 & 1 & 1 & 1 & 1 \\
\hline Ga/Dangme & $0.44(0.25,0.78)^{*}$ & $0.81(0.42,1.56)$ & $1.06(0.43,2.64)$ & $0.39(0.20,0.77)^{*}$ & $0.71(0.32,1.57)$ & $0.52(0.17,1.55)$ \\
\hline Ewe/Guan & $0.44(0.28,0.69)^{*}$ & $1.17(0.71,1.92)$ & $0.72(0.31,1.65)$ & $1.02(0.53,1.97)$ & $1.35(0.62,2.94)$ & $0.59(0.19,1.89)$ \\
\hline Mole-Dagbani & $0.19(0.11,0.30)^{*}$ & $1.19(0.76,1.88)$ & $1.59(0.84,3.02)$ & $0.79(0.31,2.03)$ & $1.11(0.42,2.92)$ & $1.30(0.29,5.67)$ \\
\hline Grussi/Gruma/ Mande & $0.39(0.23,0.68)^{*}$ & $2.31(1.38,3.88)^{*}$ & $1.88(0.88,4.03)$ & $1.17(0.44,3.10)$ & $2.28(0.87,6.011)$ & $0.95(0.22,4.22)$ \\
\hline \multicolumn{7}{|l|}{ Wealth } \\
\hline Poor & 1 & 1 & 1 & 1 & 1 & 1 \\
\hline Middle & $3.69(2.22,6.14)^{*}$ & $0.84(0.49,1.44)$ & $0.84(0.39,1.79)$ & $2.26(1.21,4.20)^{*}$ & $1.08(0.58,1.99)$ & $1.11(0.46,2.71)$ \\
\hline Rich & $3.18(2.21,4.57)^{*}$ & $0.52(0.36,0.76)^{*}$ & $0.33(0.18,0.62)^{*}$ & $1.50(0.81,2.78)$ & $0.93(0.51,1.70)$ & $0.42(0.15,1.17)$ \\
\hline \multicolumn{7}{|l|}{ Employment (past 12 months) } \\
\hline Not employed & 1 & 1 & 1 & 1 & 1 & 1 \\
\hline Employed & $0.88(0.58,1.34)$ & $1.43(0.89,2.29)$ & $2.56(1.06,6.14)^{*}$ & $1.25(0.77,2.01)$ & $1.19(0.71,2.02)$ & $2.59(1.01,6.62)^{*}$ \\
\hline
\end{tabular}

OR - Crude odds ratio; AOR - Adjusted odds ratio; Cl- Confidence Interval; vs - versus * $p<0.05$.

Regarding alcoholic beverage consumption and age, older women especially those in their thirties and forties had a higher likelihood of consuming alcoholic beverage compared to younger women in their teens and twenties. However, a woman's age was not a significant factor in determining her drinking frequency of alcoholic beverage.
This implies that a woman's age does not influence the frequency (none, once, 2-3 times, 4 or more times) at which she may consume alcoholic beverage.

It became evident that, women in the Upper West, Upper East, Central, Greater Accra, Volta and Ashanti Regions were found to have higher odds of consuming 
alcoholic beverage compared to women of the Western Region. Among these regions, women in the Upper West Region were more likely to consume alcoholic beverage. Also, a higher proportion of these women (37\%) in the region, compared to other regions, consumed alcoholic beverage $2-3$ times. The present study's finding concurs with a study by Luginaah and Dakubo [38] which suggest that inhabitants of the region increasingly consume alcoholic beverages. The aforementioned study [38] focused on a community's perception on alcohol use. According to this paper, men consume alcohol mainly for coping responses, for example, increased selfconfidence, adult status, and to cope with the various social demands. On the side of women, they seem to consume alcohol for purpose of socializing with their peers. Also, women in the region consume alcoholic beverage, perhaps, due to the insufficient sustainable means of livelihood and gainful employment. On the other hand, the region is noted with pito (locally brewed alcoholic beverage), and this too can contribute to why women reported that they consume alcohol. In addition to the pito, other alcoholic beverages are readily available to women in the region. Since the 2008 GDHS did not specify the kind of alcoholic beverage consumed by the women, the present study can conclude that some women in the region consume alcoholic beverage; which speaks little of the volume of alcoholic beverage they consume. Hence, it was not enough to also conclude in the present study that women in the Upper West Region frequently consume alcoholic beverage. By controlling for all significant background characteristics, it was revealed that women in the Upper West Region once a while consume alcoholic beverage but rather women in the Eastern Region were more likely to consume alcoholic beverage 2-3 times and those in the Greater Accra Region were more likely to consumed alcoholic beverage 4 or more times. Probably women in these regions are equally engaged in social occasions where alcoholic beverages are at their disposal or they generally have a higher tendency of consuming alcoholic beverage frequently. Longitudinal studies may be needed to better understand differences in the consumption and the drinking pattern (in terms of volume) of alcoholic beverage in the country. Also, more detailed surveys that take into account a variety of aspects of alcohol use, such as context, motivations and expectations, would be useful for improving the understanding of alcohol use in the country.

The present study further revealed that women of the Grussi, Gruma, or Mande, Ga/Dangme, and Mole-Dagbani ethnic groups commonly consumed alcoholic beverage compared to those of the Akan ethnic group. In addition, Grussi, Gruma or Mande women reported more of ever consuming alcoholic beverage. However, higher proportions of women from these ethnic groups consumed alcoholic beverage once in the past week. These findings can be attributed to the fact that these ethnic groups are found in regions that are associated with alcohol consumption; predominately, Grussi, Gruma or Mande in the Upper East Region, Mole-Dagbani in the Northern Region and $\mathrm{Ga} /$ Dangme in the Greater Accra Region. It will be worthwhile for community based studies to be conducted to assess the consumption and drinking frequency of alcoholic beverage among various homogenous groups.

Alcoholic beverage consumption has been found to be associated with economic status of an individual [43]. In the present study, women of the middle and rich wealth quintiles were less likely to consume alcoholic beverage compared to those in the poor wealth quintile. The present study's analysis reflects that most women had attained at least secondary education and this implies that they are likely to be in a higher economic status. Hence, these higher economic status women would be in a better position to make well informed decisions by not initiating the consumption of alcoholic beverage. However, when all significant factors were controlled in the multinomial regression analysis, it further showed that women of the middle wealth quintile were less likely not to have consumed alcoholic beverages. This outcome can be attributed to the educational level and better access to information that tend to inform them to drink less alcoholic beverage.

In line with results of a study by Martinez et al. [31], the present study found that women who were employed reported of consuming alcoholic beverage and were 4 or more times more likely to have consumed alcoholic beverage compared to those who were not employed. Martinez et al. [31] found out that women working for pay had a higher likelihood of being current consumers in Mauritius, Chad and Ghana. Probably, women who are involved in part-time works or who were employed in the past 12 months before the survey had a higher purchasing power for alcoholic beverage compared to those who were not employed. It can also be that this category of women tend to socialize more and in the process are more likely to consume alcoholic beverage.

The findings of this study should be considered in relation to a number of limitations. One limitation is the generalized nature in which alcohol data were collected among the women in the past week. Another is the collapsing of some variable categories that might have affected them been significant or otherwise. The study design (cross-sectional) limits the present study's ability to draw inferential interconnections between the independent variables and the consumption and drinking frequency of alcoholic beverage in the country. Other limitations associated with the present study are the inherent bias (over reporting or underreporting) in selfreport and the limited data collection time frame for the 
consumption and drinking frequency of alcoholic beverages. Beside, limited related references were used to support or refute the present study's findings. Also, the study acknowledges the lack of any quantity measure of alcohol use among women. Notwithstanding these limitations, the 2008 GDHS presents reliable alcohol related datasets that can be considered as the benchmark of the consumption and drinking frequency of alcoholic beverage surveys. Based on these limitations, the present study suggests to the Ghana Statistical Service, which is mainly responsible for conducting the GDHS, to include better measures of alcohol use in future surveys. However, the present study's findings are reflective of women's consumption and drinking frequency of alcoholic beverage in the country.

\section{Conclusions}

In the country, there was an indication of alcohol consumption among women. Women of all age groups across the regions consume a certain quantity of alcoholic beverage. It can also be concluded that, really, women do consume alcoholic beverage in the country. The study recommends that the Ministry of Health in collaboration with the media should design and rollout programmes on the maintenance of moderate consumption of alcoholic beverage among women. Also, more studies are needed to assess the consequences and reasons of drinking frequency of alcoholic beverage. To better apply interventions, it will be cost-efficient to cover regions or groups that are likely to consume alcoholic beverage. This will facilitate in drawing a comprehensive national alcohol policy in the country.

\section{Competing interests}

The authors declare that they have no competing interests.

\section{Authors' contributions}

AMTN conceived the study topic, and was involved in the study design, data analysis and drafting of the manuscript. SAT participated in drafting and review of the manuscript. Both authors read and approved the final manuscript.

\section{Acknowledgements \\ The authors acknowledge MeasureDHS for making data available for the study. We, also, acknowledge the reviewers for their comments that facilitated in enriching the content of this paper. Also, we are much grateful to the reviewers of this paper; their comments facilitated in improving the quality of the paper.}

Received: 10 September 2014 Accepted: 18 March 2015

Published online: 01 April 2015

\section{References}

1. Rehm J, Mathers C, Popova S, Thavorncharoensap M, Teerawattananon Y, Patra J. Global burden of disease and injury and economic cost attributable to alcohol use and alcohol use disorders. Lancet. 2009;373:2223-33.

2. Humeniuk RE, Henry-Edwards S, Ali RL, Poznyak V, Monteiro M. The ASSIST linked brief intervention for hazardous and harmful substance use: manual for use in primary care. Geneva: World Health Organization (WHO), Department of Mental Health and Substance Abuse; 2010.
3. Lim S, Vos T, Flaxman A, Danaei G, Shibuya K, et al. A comparative risk assessment of the burden of disease and injury attributable to 67 risk factors and risk factor clusters in 21 regions, 1990-2010: a systematic analysis for the Global Burden of Disease Study 2010. Lancet. 2012;380:2224-60.

4. Abbey A, Alcohol-related sexual assault. A common problem among college students. J Stud Alcohol Suppl. 2002;14:118-28.

5. Baan R, Straif K, Grosse Y, Secretan B, El Ghissassi F, Bouvard V, et al. Carcinogenicity of alcoholic beverages. Lancet Oncol. 2007;8:292-3.

6. Booth $B M$, Feng $W$. The impact of drinking and drinking consequences on short-term employment outcomes in at-risk drinkers in six southern states. J Behav Health Serv Res. 2002;29(2):157-66.

7. Kloner RA, Rezkalla SH. To drink or not to drink? That is the question. Circulation. 2007;116:1306-17

8. International Centre for Alcohol Policies (ICAP). Standard drinks: defining a standard drink. 2014. http://www.icap.org/PolicyTools/ICAPBlueBook/ BlueBookModules/20StandardDrinks/tabid/161/Default.aspx.

9. National Institute on Alcohol Abuse and Alcoholism (NIAAA). Alcohol: a women's health issue. 2008. http://pubs.niaaa.nih.gov/publications/ brochurewomen/women.htm.

10. International Centre for Alcohol Policies (ICAP). International drinking guidelines. 2010. http://www.icap.org/table/Internationaldrinkingguidelines

11. National Institute on Alcohol Abuse and Alcoholism. Overview of alcohol consumption: drinking levels defined. 2014. http://www.niaaa.nih.gov/ alcohol-health/overview-alcohol-consumption/moderate-binge-drinking.

12. Department of Health. South African guidelines for healthy eating: for adults and children over the age of seven years. Pretoria, South Africa: Nutrition Directorate: 2004.

13. Pearson TA. Alcohol and heart disease. Circulation. 1996;94:3023-5.

14. Simons LA, Mccallum J, Friedlander Y, Ortiz M, Simons J. Moderate alcohol intake is associated with survival in the elderly: the Dubbo study. Med J Aust. 2000;173(3):121-3.

15. Sacco RL, Elkind M, Boden-Albala B, Lin IF, Kargman DE, Hauser WA, et al. The protective effect of moderate alcohol consumption on ischemic stroke. J Am Med Assoc. 1999;281:53-60

16. Hines LM, Rimm EB. Moderate alcohol consumption and coronary heart disease: a review. Postgrad Health J. 2001;77:747-52.

17. Suzuki K, Elkind MSV, Boden-Albala B, Jin Z, Berry G, Di Tullo MR, et al. Moderate alcohol consumption is associated with better endothelial function: a cross sectional study. BMC Cardiovasc Discorder. 2009;9:8

18. Hanson DJ. Should doctors recommend drinking alcohol to patients for good health. 2013 http://www2.potsdam.edu/alcohol/Healthlssues/ 1122661918.html\#.U_aL22Mr7Gg.

19. Djousse L, Gaziano JM. Alcohol consumption and heart failure. Curr Atheroscler Rep. 2008;10(2):117-20.

20. American Gastroenterological Association. "Alcohol consumption habits may threaten GI Health". ScienceDaily 2006. http://www.sciencedaily.com/ releases/2006/05/060522235250.htm.

21. Suter PM. Is alcohol consumption a risk factor for weight gain and obesity? Crit Rev Clin Lab Sci. 2005:42(3):197-227.

22. Baik I, Shin C. Prospective study of alcohol consumption and metabolic syndrome. Am J Clin Nutr. 2008;87(5):1455-63.

23. Nakanishi N, Suzuki K, Tatara K. Alcohol consumption and risk for development of impaired fasting glucose or type 2 diabetes in middle-aged Japanese Men. Diabetes Care. 2003;26(1):48-54.

24. Juhl M, Nyboe Andersen AM, Gronbaek M, Olsen J. Moderate alcohol consumption and waiting time to pregnancy. Hum Reprod. 2001;16:2705-9.

25. Sara LW, Kevan RP, Alan C, Jonathan ES, Robert CA, Steven JC. Alcohol consumption and 5-year onset of chronic kidney disease: the AusDiab study. Nephrol Dial Transplant. 2009;24(8):2464-72.

26. Kenigsberg B. How does alcohol affect hypertension? Clinical correlations: The NYU Langone Online Journal of Medicine 2011. http://www. clinicalcorrelations.org/? $\mathrm{p}=4534$

27. O'Shea RS, Dasarathy S, McCullough AJ, et al. AASLD practice guidelines: alcoholic liver disease. Hepatology. 2010;51(1):307-28

28. Schuppan D, Afdhal NH. Liver cirrhosis. Lancet. 2008;371:838-51.

29. Partnership for Maternal, Newborn and Child Health (PMNCH). PMNCH knowledge summary number 15: non-communicable diseases - harmful use of alcohol. Geneva: WHO; 2011.

30. WHO. Partnership for maternal, newborn and child health: knowledge summary-non-communicable diseases. No. 15. Geneva, Switzerland: World Health Organization; 2011. 
31. Martinez P, Roislien J, Naidoo N, Clausen T. Alcohol abstinence and drinking among African women: data from the World Health Surveys. BMC Public Health. 2011;11:160.

32. Modern Ghana. National acohol draft policy launched. 2009. http://www. modernghana.com/news/255087/1/national-alcohol-draft-policy-launched.html.

33. Ghana Statistical Service [GSS], Ministry of Health [MOH] \& ICF Macro. Ghana Demographic and Health Survey 2008. Accra: GSS, MOH and ICF Macro; 2009.

34. Food and Drugs Board (FDB). Food and Drugs Board slams alcoholic ads. 2007 http://www.modernghana.com/news/143142/1/fdb-slams-alcoholic-ads.html.

35. Brako-Powers A. Feminine face of alcohol in Ghana; 2011. Myjoyonline. http://opinion.myjoyonline.com/pages/feature/201109/73421.php.

36. World Health Organization (WHO): Global Status Report on Alcohol and Health. WHO, Geneva; 2011.

37. Owusu SA. Culture shock and behavioural trends in Ghana. 2011. http://www ghanaweb.com/GhanaHomePage/NewsArchive/artikel.php?ID=210595.

38. Luginaah I, Dakubo C. Consumption and impacts of local brewed alcohol (akpeteshie) in the Upper West Region of Ghana: a public health tragedy. Soc Sci Med. 2003;57(9):1747-60.

39. Adusi $Y$, Bonney AA, Antwi GD. Where, when and what type of alcohol do pregnant women drink? Ghana Med J. 2013;47(1):35-9.

40. Agoabasa FA. Alcoholism among women and its impact on the socio-economic development of Ghana: a case study of Sandema [unpublished dissertation]. Accra, Ghana: Department of Business Administration, Ashesi University College; 2012.

41. Ghana Statistical Service (GSS). 2000 Population and Housing Census: final results. Accra: GSS; 2012

42. National Institutes of Health $(\mathrm{N} I \mathrm{H})$. Rethinking Drinking: Alcohol and your health. National Institute on Alcohol Abuse and Alcoholism. NIH Publication No. 13-3770; 2010. www.rethinkingdrinking.niaaa.nih.gov.

43. Elgar FJ, Roberts C, Parry-Langdon N, Boyce W. Income inequality and alcohol use: a multilevel analysis of drinking and drunkenness in adolescents in 34 countries. Eur J Pub Health. 2005;15(3):245-50.

\section{Submit your next manuscript to BioMed Central and take full advantage of:}

- Convenient online submission

- Thorough peer review

- No space constraints or color figure charges

- Immediate publication on acceptance

- Inclusion in PubMed, CAS, Scopus and Google Scholar

- Research which is freely available for redistribution 